\title{
Chapter 36 \\ Determining the Order of Resistance \\ Genes Qsng-3BS, Fhb1 and Sr2 and Combining Them in Coupling on Wheat Chromosome 3BS
}

\author{
Rima Thapa, Gina Brown-Guedira, Herbert W. Ohm, \\ Kiersten Wise, and Stephen B. Goodwin
}

\begin{abstract}
A variety of diseases of wheat (Triticum aestivum L.) occurs every year in the U.S. leading to significant grain yield losses. Stagonospora nodorum blotch (SNB), fusarium head blight (FHB) and stem rust (SR) are caused by the fungi Stagonospora nodorum, Fusarium graminearum and Puccinia graminis, respectively. These diseases penalize both grain yield and quality. Three resistance factors, Qsng.sfr-3BS, Fhbl and Sr2 conferring resistance, respectively, to SNB, FHB and $\mathrm{SR}$, each from a unique donor wheat parent line, have been mapped to chromosome $3 \mathrm{BS}$ of wheat and are believed to be closely linked. Based on previously published analyses, $S r 2$ is on the distal end, Fhbl is on the proximal end and Qsng.sfr- $3 B S$ is in the middle of $S r 2$ and $F h b 1$ in the 3BS wheat genome. Thus, the objectives of this project are to determine the gene order of Qsng.sfr-3BS, Fhbl and Sr2, in a linkage block on chromosome $3 \mathrm{BS}$ and combining them in coupling. The linkage relationships were determined through analysis of a three-way cross between parental lines Arina, Alsen and Ocoroni86, containing the resistance genes Qsng.sfr-3BS, Fhbl and $S r 2$, respectively. A total of $1,600 \mathrm{~F}_{2}$ plants was screened, along with the parental lines, using KASPar genotyping technology via single-nucleotide polymorphism markers to identify the recombinant progeny. Phenotypic screening for SNB was performed on the entire $\mathrm{F}_{2}$ population. Knowing the positional order of these
\end{abstract}

\footnotetext{
R. Thapa $(\bowtie) \bullet$ H.W. Ohm

Department of Agronomy, Purdue University, West Lafayette, IN 47907, USA

e-mail: rthapa@purdue.edu

G. Brown-Guedira

USDA-ARS, Crop Science Department, North Carolina State University,

Box 7620, Raleigh, NC 27695, USA

K. Wise

Department of Botany and Plant Pathology, Purdue University,

915 West State Street, West Lafayette, IN 47907-2054, USA
}

S.B. Goodwin

Crop Production and Pest Control Research Unit, USDA-ARS, Purdue University,

915 West State Street, West Lafayette, IN 47907-2054, USA 
resistance genes will enable the development of a wheat line with three genes in coupling to provide durable and broad-spectrum resistance against three major diseases of wheat.

\section{Introduction}

Stagonospora nodorum blotch (SNB), fusarium head blight (FHB) and stem rust (SR) of wheat are caused by the fungi Stagonospora nodorum, Fusarium graminearum and Puccinia graminis, respectively. Each of these diseases can cause yield losses up to $50 \%$ or more during severe epidemics and when environmental conditions are favorable (Chester 1943; Roefls 1978; Stakman and Harrar 1957; Wicki et al. 1999). In addition to yield reduction, FHB reduces quality due to production of a mycotoxin called vomitoxin (deoxynivalenol) produced by $F$. graminearum (Bai et al. 2001; Gilbert and Tekauz 2000), which is harmful to both humans and livestock. FHB also reduces test weight and lowers market grade. Thus, FHB is one of the most feared fungal diseases of wheat because an entire crop can be rejected for human consumption due to mycotoxin contamination. SR has a capacity of destroying millions of hectares of healthy, high-yielding wheat in less than a month by reducing fields to a mass of bare stalks supporting only small, shriveled grains by harvest time (Singh et al. 2008). There have been several epidemics of SR during the past 80 years that have reduced the yield by $50 \%$ in the Great Plains (Chester 1943; Roefls 1978; Stakman and Harrar 1957). SNB is one of the major foliar and glume diseases of wheat and the most yield loss occurs when the flag leaf and the two leaves below the flag leaf become infected by the time the wheat flowers in late May.

The objective of this study is to find the gene order of Fhb1, Sr2 and Qsng.sfr$3 B S$ with the long-term goal of combining them in a linkage block on wheat chromosome 3BS. The first objective was achieved by crossing three unique parental lines with resistance genes $F h b 1, S r 2$ and Qsng.sfr-3BS to combine them into one background. An $\mathrm{F}_{2}$ population segregating for all three genes was genotyped with single-nucleotide polymorphism (SNP) markers to validate the presence of markers linked to the resistance genes and also to determine the gene order. The $\mathrm{F}_{2}$ population was also phenotyped for level of resistance to SNB.

\section{Materials and Methods}

The mapping population consisted of $1,600 \mathrm{~F}_{2}$ progeny derived from a three-way cross between wheat cultivars Arina, Alsen and Ocoroni86 providing the resistance genes Qsng.sfr-3BS, Fhbl, and $S r 2$, respectively. The $\mathrm{F}_{2}$ population, its parents and cultivar Chinese Spring as negative control, were planted in January, 2013 in a greenhouse at Purdue University in West Lafayette, Indiana, U.S.A.. The primary 
spike after spike emergence (Feekes growth stage 10.3) of an adult plant of every $F_{2}$ progeny and the parental lines was phenotyped in a greenhouse using an isolate of $S$. nodorum obtained from an infected wheat field in Indiana. The disease severity scores were recorded 21 days after inoculation on a $0-9$ scale. The $F_{2}$ plants were screened along with the parental lines using KASPar genotyping technology to identify the recombinant plants. Single-nucleotide polymorphism markers were utilized to identify the recombinants, determine the gene order, to make the genetic map and for quantitative trait loci (QTL) analysis. Linkage analysis was performed and the map was made with JoinMap 3.0.

\section{Results and Discussion}

Phenotyping of all $1,600 \mathrm{~F}_{2}$ progeny was performed and the results utilized the full range of the 0-9 disease-rating scale. The frequency distribution of the percent diseased glume tissue of $\mathrm{F}_{2}$ progeny derived from the three-way cross of Arina (containing the Qsng.sfr-3BS SNB resistance QTL), Alsen and Ocoroni86 inoculated with a field isolate of $S$. nodorum in a greenhouse showed a continuous distribution with a skew towards susceptibility, indicating the presence of one or more QTL for resistance (data not shown).

The preliminary genetic linkage map suggests that the stem rust resistance gene $(S r 2)$ is the most distal, the fusarium head blight resistance gene ( $F h b 1)$ is proximal, and with the $S$. nodorum resistance gene (Qsng.sfr-3BS) is between $S r 2$ and $F h b 1$ on chromosome 3BS of wheat (Fig. 36.1). This result supports our hypothesis made

Fig. 36.1 Preliminary linkage map of wheat chromosome 3BS for markers SNP 1, SNP 2, SNP 3, SNP 4 , and SNP 5 in the F2 population of 1,600 individuals derived from a three-way cross of wheat cultivars Arina, Alsen and Ocoroni86. The map was generated with JoinMap 3.0 at $\mathrm{LOD}=9.0$. Numbers to the left of the vertical bar indicate the total distance in centimorgans, and positions of mapped markers are indicated on the right. The approximate positions of disease resistance genes are indicated by brackets

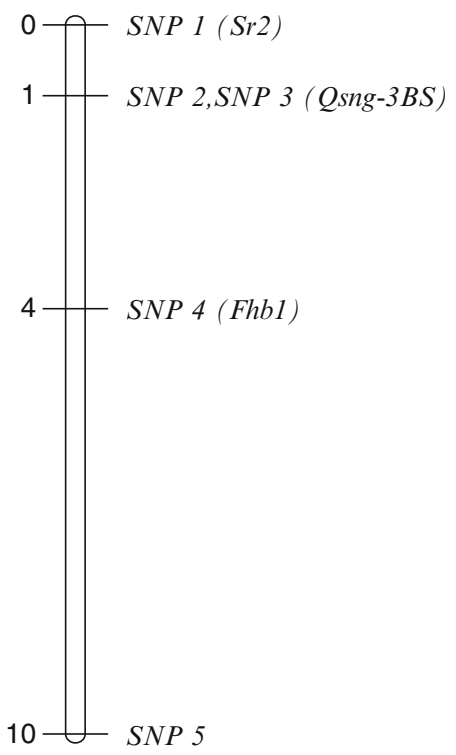


from analyses of previously published marker positions. Knowing the correct order and relative distance between the three resistance genes indicates how a linkage block can be created. Markers for $S r 2$ and Qsng.sfr-3BS were placed $1 \mathrm{cM}$ apart in our mapping population while the predictive marker for $F h b 1$ was located $4 \mathrm{cM}$ proximal from $S r 2$. Thus, recombinant plants having both $S r 2$ and Qsng.sfr-3BS were recovered at a lower frequency than plants having Fhbl and Qsng.sfr-3BS in coupling. These plants having two resistance genes in coupling will be used in further crosses to obtain recombinants having all three resistance genes in a linkage block. The tight linkage between Sr2, Qsng.sfr-3BS, and Fhbl indicates that it should be easy to maintain this linkage block in a breeding program.

Open Access This chapter is distributed under the terms of the Creative Commons Attribution Noncommercial License, which permits any noncommercial use, distribution, and reproduction in any medium, provided the original author(s) and source are credited.

\section{References}

Bai GH, Plattner R, Desjardins A, Kolb FL (2001) Resistance to Fusarium head blight and deoxynivalenol accumulation in wheat. Plant Breed 120:1-6

Chester KS (1943) The decisive influence of late winter wheat on wheat leaf rust epiphytotics. Plant Dis Rep Suppl 143:133-44

Gilbert A, Tekauz A (2000) Review: recent developments in research in fusarium head blight of wheat in Canada. Can J Plant Pathol 22:1-8

Roefls AP (1978) Estimated losses caused by rust in small grain cereals in the United States-1918-76, vol 1363, Miscellaneous publication (United States. Department of Agriculture). U.S. Department of Agriculture, Agricultural Research Service, Washington, DC, $85 \mathrm{pp}$

Singh RP, Hodson DP, Huerta-Espino J, Jin Y, Njau P et al (2008) Will stem rust destroy the world's wheat crop? Adv Agron 98:271-309

Stakman EC, Harrar JC (1957) Principles of plant pathology. Ronald Press, New York, 553 pp

Wicki W, Winzeler M, Schmid JE, Stamp P, Messmer M (1999) Inheritance of resistance to leaf and glume blotch caused by Septoria nodorum Berk. in winter wheat. Theor Appl Genet 99:1265-1272 